\title{
ANALYZING MIXING QUALITY IN A T-SHAPED MICROMIXER FOR DIFFERENT FLUIDS PROPERTIES THROUGH NUMERICAL SIMULATION
}

\author{
A. S. Lobasov, A. V. Minakov \\ Siberian Federal University \\ Institute of Thermophysics SB RAS, Novosibirsk \\ E-mail: perpetuityrs@mail.ru
}

Microreaction engineering enables new strategies in process intensification. A precise analysis of local mass transfer and hydrodynamics in micromixers for different flow regimes are strongly needed for a complete understanding the processes occurring. One of the simplest in the manufacture, but at the same time, quite effective T-shaped micromixer was used for numerical investigation and analyzing the mixing quality and flow regimes as well as the influence of different fluids properties on this parameters. It was numerically revealed that the viscosities and the densities, as well as the initial temperatures and the rheology of mixing fluids have significant effects on the flow regimes and the mixing efficiency of two fluids. In this study viscosities and densities ratios of mixing fluids ranged from 1 to 2 ; the coefficient $n$ in power-law model of non-Newtonian fluids ranged from 0.3 to 1 ; the initial temperatures difference of two fluids was varied up to $40^{\circ} \mathrm{C}$. Mixture components concentration as well as pressure and velocity fields distribution in the micromixer was obtained. The dependence of fluids mixing efficiency and the pressure drop, as well as a map of flow regimes and mixing modes on the Reynolds number and properties of miscible fluids was numerically established.

Keywords: Hydrodynamics, numerical simulation, T-shaped micromixer, microagitator, mixing, viscosity, temperature, rheology, pressure drop.

\section{INTRODUCTION}

The miniaturization of technological processes is being actively promoted in recent years in the chemical industry, and thus micromechanics has become a rapidly developing and promising research area. Microchannel devices are widely used in various fields of science and technology as microreactors, microscale heat exchangers, micromixers, etc. Many studies have noted that the use of microdevices allows significantly enhancing the physicochemical processes in comparison to classical space consuming reactors [1-3]. It can be confidently predicted that the situation in the chemical laboratories will change radically in the near future 
toward a significant improvement in the productivity and efficiency of synthesis processes through a substantial miniaturization of instruments and apparatuses. Control of pressure, temperature, reaction time and flow velocities in reactors with small volume is carried out much easier and more efficiently. Thus, the main undeniable advantages of microreactor microsystems are safety of carrying out highly exothermic reactions and working with toxic or explosive reagents, carrying out reactions in supercritical conditions and significantly reducing the cost of research, as well as implementation and scaling of chemical processes.

However, despite the obvious benefits of microchannel technology, there are a number of specific problems. Most microchannel devices that used in chemistry and biology require fast and effective mixing of substances [1-5]. At the same time, the flow in microchannels is predominantly laminar and mixing occurs through diffusion, and thus very slowly. Therefore, the design and optimization of micromixers with the shortest mixing time is an actual problem in the development of microchannel devices.

The T-shaped micromixers (Fig.1) are the simplest in the manufacture in terms of their underlying geometry. The first study of T-shaped micromixer [6] shown very high stirring rates obtaining in such configuration of micromixers. Flow regimes at low Reynolds numbers have been systematically studied previously in many works [6-8]. Apparently, first studies of fluids mixing processes in T-shaped micromixer at moderate Reynolds numbers were conducted in [9]. The effective mixing rate in the T-shaped micromixer at a certain inlet flow velocities was obtained experimentally for the first time in that work. The most comprehensive experimental study of mixing in T-shaped micromixer at moderate Reynolds numbers (100-400) was carried out in [10].

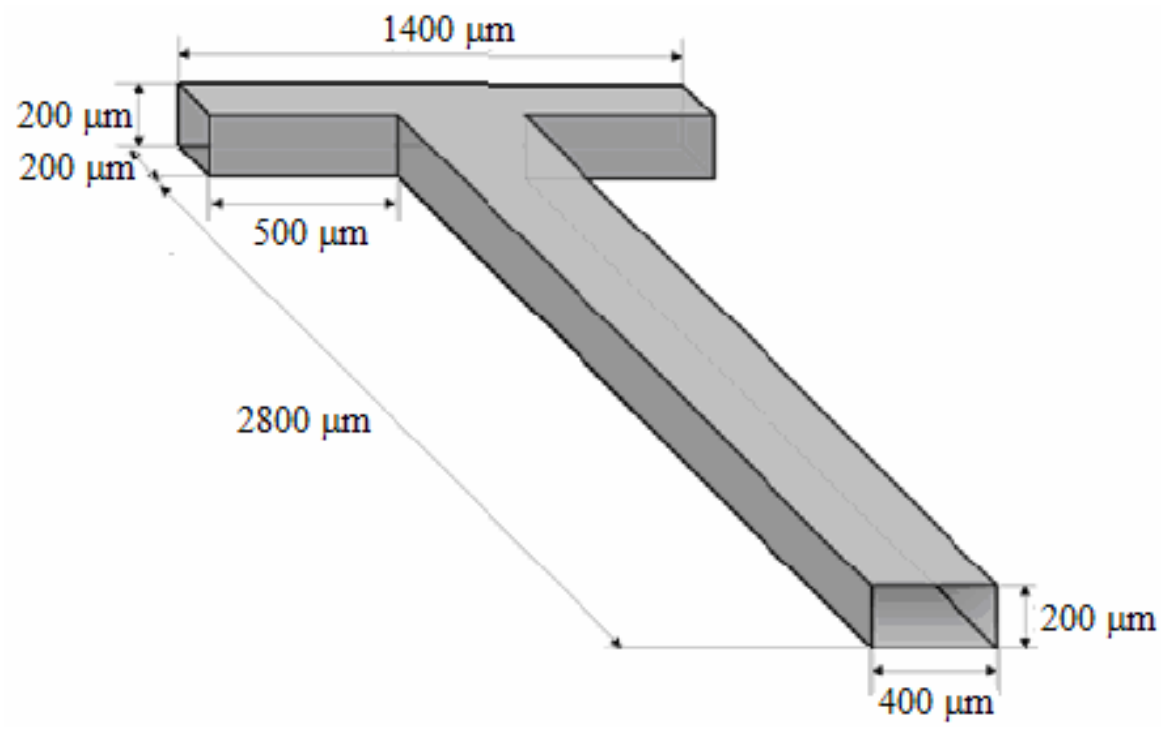

Figure 1. Geometric configuration of the task 
A number of experimental and theoretical works [11-14] should be also mentioned. They present calculations of some flow regimes in T-shaped micromixers with different cross sections. The existence of a certain critical Reynolds number, at which Dean vortices in the Tshaped micromixer lose their symmetry was shown experimentally.

A more detailed study of this effect was carried out in a series of works [15-17]. Flow regimes and mixing modes in the water-water system in the T-shaped micromixer were investigated within a wide range of Reynolds number from 1 to 1000 , using numerical simulation, micro Particle Image Velocimetry ( $\mu$-PIV), and micro Laser Induced Fluorescence $(\mu$-LIF) measurements.

The flow structure and its influence on mixing mode was investigated, five different flow regimes were revealed, and dependences of friction coefficient and mixing efficiency on the Reynolds number were obtained. Dramatic increase in mixing efficiency at transition from symmetric to asymmetric mode for stationary flow regime was noted. The laminar-turbulent transition area was investigated and good qualitative and quantitative agreement with the results of field experiments was obtained.

A systematic study of the viscosity effect on mixing modes in the T-shaped micromixer was carried out in [18], where the significant effect of viscosities ratio on the flow structure before and after the transition from symmetric to asymmetric flow regimes was shown. The self-similar behavior of the asymmetric flow regime is established.

Also a comprehensive investigation of mixing of binary fluids with compositiondependent viscosity in a T-shaped micro-device was carried out in [19]. In that study, it was shown that in the case of a positive fluidity of mixing, the onset of the engulfment regime is accompanied by a sharp increase of the degree of mixing, with the critical Re decreasing as the fluidity of mixing increases. This research was developed in [20], where unsteady mixing of binary liquid mixtures with composition-dependent viscosity was studied. It was shown that a viscous layer forms at the confluence of the inlet flows when the viscosity of the mixture is larger than that of the pure components. It tends to keep the two streams separated, resulting in a shift of the engulfment regime towards larger Reynolds numbers.

Water-ethanol mixing in T-shaped microdevices was investigated numerically in [21] and experimentally in [22] taking into account both density and viscosity variations. The results were compared with studies of water-water mixing. In both research works, it was shown that at smaller Reynolds number there is no vortex formation for either cases, and at larger Reynolds numbers, mixing of water and ethanol may take considerably longer, as the onset of engulfment is retarded and occurs at larger Reynolds number. 
In addition to micromixing of Newtonian fluids, the problem of non-Newtonian fluids mixing (blood, polymer solutions, suspensions) is relevant as well. A study of non-Newtonian fluids mixing in serpentine and T-shaped micromixers was carried out in [23]. The Reynolds number ranged from $10^{-2}$ to $10^{2}$. A blood, whose viscosity was given by Casson and CarreauYasuda non-Newtonian blood viscosity models, served as a non-Newtonian fluid. It was shown that the efficiency of mixing in T-shaped mixer decreases with the increase in the Reynolds number due to the corresponding increase in the Peclet number. In the serpentine micromixer the mixing efficiency originally decreased and then sharply increased upon reaching the region of transition from symmetric to asymmetric mode at stationary flow.

The effect of non-Newtonian fluid characteristics, specifically the effect of shearthinning on the critical Reynolds number through the use of the power-law model in the power-law index range from 0.5 to 1 was investigated in [24]. It was shown as a result that, depending on the exact choice of Reynolds number, it would be possible to claim that shearthinning either promotes or inhibits the bifurcation to asymmetric flow.

Thus, flow regimes depending on the Reynolds number were investigated in many works [18-21,24-29]. At that, quite interesting hydrodynamic phenomenon, named flow reversal or engulfment flow regime upon reaching the critical Reynolds number was observed in the T-shaped mixer. This critical Reynolds number differs for different mixtures, also it strongly depends on the channel geometry, e.g., for water-water mixing in the T-channel, shown in Fig. 1 the critical Reynolds number is equal to 140-150. The aim of the present work is to carry out systematic investigation of the effects of miscible fluids properties on the flow regimes and the critical Reynolds number in the T-shaped micromixer.

\section{MATHEMATICAL MODEL AND NUMERICAL ALGORITHM}

Incompressible flows of multi-component and generally non-Newtonian fluids, which are described using a hydrodynamic approach based on the solution of the Navier-Stokes equations were considered in this work. Currently, in numerous experiments was shown that such description for fluids works well for the channels sizes up to 1 micron.

In general, the Navier-Stokes equations system has the following form:

$$
\begin{gathered}
\frac{\partial \rho}{\partial t}+\nabla(\rho \mathbf{v})=0 \\
\frac{\partial \rho \mathbf{v}}{\partial t}+\nabla(\rho \mathbf{v v})=-\nabla p+\nabla \mathbf{T}+\rho \mathbf{g}
\end{gathered}
$$

Here $\rho$ is the fluid density, $p$ is the pressure, $\mathbf{v}$ is the velocity, and $\mathbf{T}$ is the tensor of viscous stresses, $\mathbf{g}$ is the gravity vector. 
The widely known approach [30,31], in which the medium is considered as a nonlinear viscous fluid characterized by the effective fluid viscosity $\mu(\dot{\gamma})$ depending in general on shear rate, was used for the simulation of non-Newtonian flows. At that, the viscous tension tensor is defined as $\mathbf{T}=\mu \varepsilon$, while components of the strain velocity tensor $\boldsymbol{\varepsilon}$ can be written as:

$$
\boldsymbol{\varepsilon}_{i j}=\frac{\partial u_{i}}{\partial x_{j}}+\frac{\partial u_{j}}{\partial x_{i}} .
$$

Shear rate $\dot{\gamma}$ is the second invariant of the strain velocity tensor:

$$
\dot{\gamma}=\sqrt{\frac{1}{2} \sum_{i \neq j} \boldsymbol{\varepsilon}_{i} \boldsymbol{\varepsilon}_{j}} .
$$

The considered medium may be both Newtonian viscous fluid and non-Newtonian viscoplastic fluid, whose behavior is described by the most common "power-law" rheological fluid model.

The effective viscosity depending on the rheology of the medium is given by:

$\mu(\dot{\gamma})=k$ for Newtonian medium ( $k$ is the molecular viscosity of the fluid),

$\mu(\dot{\gamma})=k \dot{\gamma}^{n-1}$ for the power-law model, where $n$ and $k$ are coefficients of the model.

The effective viscosity of the mixture is determined through the mass fraction of its components $f$ and effective viscosities of pure components $\mu_{1,2}$ :

$$
\mu=f \mu_{1}+(1-f) \mu_{2}
$$

The density of the mixture is expressed in terms of the mass concentrations $f$ of flow components and partial densities $\rho_{1,2}$ in the following way:

$$
\frac{1}{\rho}=\frac{f}{\rho_{1}}+\frac{(1-f)}{\rho_{2}}
$$

Here the evolution of mass concentrations is determined by the equation:

$$
\frac{\partial \rho f}{\partial t}+\nabla(\rho f \mathbf{v})=\nabla(\rho D \nabla f)
$$

where $D$ is the diffusion coefficient.

To solve the above described equations system, oFlow software package for computational fluid dynamics was used. A detailed description of the numerical algorithm of this software tool is given in [31]. Here will be noted the main points of numerical technique. The difference analogue of the convective-diffusion equations (1) and (2) is determined using the finite volume method [32-34] for structured multi-block grids. In this case, the resulting scheme is automatically conservative. The method consists in splitting the computational 
domain into control volumes and integrating the original conservation equations for each control volume to obtain finite-difference equations. Approximation of convective terms of transfer equations (1) and (2) is carried out using a second order counterflow QUICK [35] and TVD [34] schemes, respectively. To approximate the unsteady terms of hydrodynamics equations a second order implicit scheme was applied. Diffusion fluxes and source terms were approximated by finite-volume analogues of the central-difference correlations with the second order of accuracy. The relationship between the velocity and pressure fields, ensuring the fulfillment of the continuity equation, was implemented by means of the SIMPLEC procedure for combined grids [32]. Rhi-Chow approach, consisting in the introduction of monotonizator into the equation for the pressure correction [36] was used to eliminate the pressure field oscillations. Obtained difference equations resulting from discretization of the original system of differential equations were solved by iterative method using algebraic multigrid solver [37].

Three-block grid consisting of 9.5 million grid nodes was used for calculations in this work. Preliminary calculations have shown that this level of grid particularization is acceptable in terms of calculation accuracy. The constant fluid flow rate with steady-state velocity profile was set at the inlets of micromixer. Neumann conditions, meaning equivalent to zero the derivative of all scalar quantities normal to the outlet surface were set at the outlet of the mixing channel. The walls of the mixer were considered as insulated. For the velocity vector components, no-slip condition was taken as boundary condition on the channels walls. The applicability of this type of boundary conditions for channels sizes up to $50 \mu \mathrm{m}$ has been demonstrated in $[1,8,16,17]$.

The channel dimensions are shown in Fig. 1. The channel thickness is $200 \mu \mathrm{m}$, while the width of its narrow and wide parts is $200 \mu \mathrm{m}$ and $400 \mu \mathrm{m}$, respectively.

The pressure drop between one of the mixer inlets and the outlet, as well as mixing efficiency were determined in this study. The mixing efficiency is usually quantified using the parameter $M=1-\left(\sigma / \sigma_{0}\right)^{0.5}$, where $\sigma=V^{-1} \int_{V}(f-\langle f\rangle)^{2} d V$ is the root-mean-square deviation of the mass fraction of mixture component $f$ from its average value $\langle f\rangle, \sigma_{0}=\langle f\rangle \cdot(1-\langle f\rangle)$ is the maximum root-mean-square deviation, and $V$ is the volume of the computational domain.

\section{RESULTS OF NUMERICAL SIMULATION}

Mixing of fluids with similar properties. First, mixing of two Newtonian fluids with similar properties was considered. Pure water was supplied to mixer through one of the inlets. 
Water, tinted with rhodamine was supplied through another inlet with the similar flow rate. The density of both fluids was $1000 \mathrm{~kg} / \mathrm{m}^{3}$, the viscosity was $0.001 \mathrm{~Pa} \cdot \mathrm{s}$, the dye diffusion coefficient in water was $D=2.63 \times 10^{-10} \mathrm{~m}^{2} / \mathrm{s}$. As noted above, a detailed study of flow regimes and mixing of water with water, dyed with rhodamine, was conducted in [15-17]. These modes will be described here briefly just for better understanding. The change in the flow regimes in the microchannel is characterized by the Reynolds number, which is defined as follows:

$$
\operatorname{Re}=\frac{\rho U d_{h}}{\mu}
$$

where $U=Q /\left(2 \rho H^{2}\right)=Q_{i n} /\left(\rho H^{2}\right)$ is the mean flow velocity in the mixing channel, $H=200 \mu \mathrm{m}$ is the channel height, $d_{h}=267 \mu \mathrm{m}$ is the hydraulic diameter.

The Reynolds number equal to 1 corresponds to creeping vortex-free flow accompanied by a weak mixing of dye. The increase in Reynolds number in the mixer leads to the development of a pair of symmetrical horseshoe vortices, which are formed near the left end wall of the mixer and propagate into the mixing channel, where they gradually decay. Horseshoe vortices appear due to the development of the secondary flows that generated owing to centrifugal force associated with the flow rotation. Such vortices are called Dean vortices. Each such horseshoe vortex located in the borders of corresponding fluids and didn't cross the interface between miscible fluids. Therefore, the interface between two media remains almost flat.

Very intriguing reconstruction of the flow regime is observed with increase the Reynolds number. Starting from the Reynolds number equal to about 145, pair of horseshoe vortices turn at an angle of $30^{\circ}$ in relation to the central longitudinal plane of the mixer. The mixing pattern in the beginning of the cross-section of the mixing channel resembles a Kelvin-Helmholtz instability form. This flow reversal causes a gradual attenuation of one horseshoe vortex branch, and increase the intensity of the other one. As a result, two intense vortices with the similar swirl are formed in the mixing channel. At that, the flow remains stationary! The intensity of vortices in asymmetric flow regime also called "engulfment flow regime" increases substantially and they stretch along the mixer channel down to the outlet. The presence of flow swirling in the mixing channel leads to the formation of the layered Sshape structure of miscible fluids. The interface of miscible fluids in such layered structure is very well developed that leads to a sharp increase in the mixing efficiency. When flow changing from the symmetric flow regime $(\operatorname{Re}<145)$ to the engulfment flow regime $(\operatorname{Re}>145)$ the mixing efficiency increases more than 25 times. 
The dependence of the mixing efficiency on the Reynolds number is shown in Fig. 2a (curve 1). In this work the mixing efficiency is computed with a very small step in the Reynolds number. It is established that the transition from symmetric to asymmetric flow regime occurs almost abruptly. Thus, in particular, the symmetric mode of flow is still observed at the Reynolds number equal to 144 , while at 144.5 the flow became engulfment. Interestingly that such a significant alteration of the flow regime has no effect on the pressure drop between the mixer inlet and outlet (Fig. 2b, curve 1).

The described above vortex flow structure exists in a stationary state within the range of Reynolds numbers from 145 to about 240 doesn't undergo significant changes. At larger Reynolds numbers the flow ceases to be stationary. Within the range $(240<\operatorname{Re}<400)$ the flow can be considered periodic. At Reynolds numbers greater than 600 , the flow in the channel becomes turbulent [16]. Between these two regimes there is transition flow regime, which can be called an unsteady quasiperiodic, it is neither periodic nor turbulent. For unsteady regimes, the mixing efficiency was evaluated by time-averaging of the obtained value. These numerical results are in good correspondence with most comprehensive experimental data of mixing in T-shaped micromixer at moderate Reynolds numbers, mentioned above [10]. E.g. the comparison of numerical and experimental dye concentration contours in the longitudinal and transverse sections of the mixer at $\operatorname{Re}=186$ shown in Fig. 3.

Effect of density. The density effect on flow regimes in the micromixer was studied. This series of calculations differed from the previous one by the fact that the density of one of the miscible fluids was varied. Water, tinted with rhodamine was supplied through one of the mixer inlets similarly to the previous experiments. Fluid with viscosity and diffusion coefficient similar to those for pure water, but changed density was supplied at the same flow rate through another inlet of the mixer. Three series of calculations with densities of $1.25,1.5$ and 2 times greater than the density of water were performed. The density of water at room temperature $\rho=1000 \mathrm{~kg} / \mathrm{m}^{3}$ was used to determine the Reynolds number. First, a series of calculations under zero gravity conditions $\left(g=0 \mathrm{~m} / \mathrm{s}^{2}\right)$ was carried out.

Increasing the density of one of the miscible fluids leads to significant change in flow pattern. For very small $(<10)$ Reynolds numbers the flow and mixing structure of fluids with different densities in general is similar to the flow of two identical fluids. Therefore, the mixing efficiency in this regime slightly depends on the densities of fluids (see Fig. 2a). The increase of Reynolds number in the channel causes formation of vortices, and the difference in densities begins to affect the mixing. Dye concentration contours corresponding to Reynolds number equal to 120 and different densities ratios are shown in Fig. 4. It is clear that 
in contrast to the case of equal densities of miscible fluids, the interface between fluids is not flat. Fluid with a higher density pushed a less dense fluid from channel walls and somewhat envelops it. This is clearly seen in Figs. 4b,4c. When fluids densities ratio increased this effect is amplified that results in increase of miscible fluids interface area and consequently the enhancement of the mixing efficiency in this regime (see Fig. 2a).

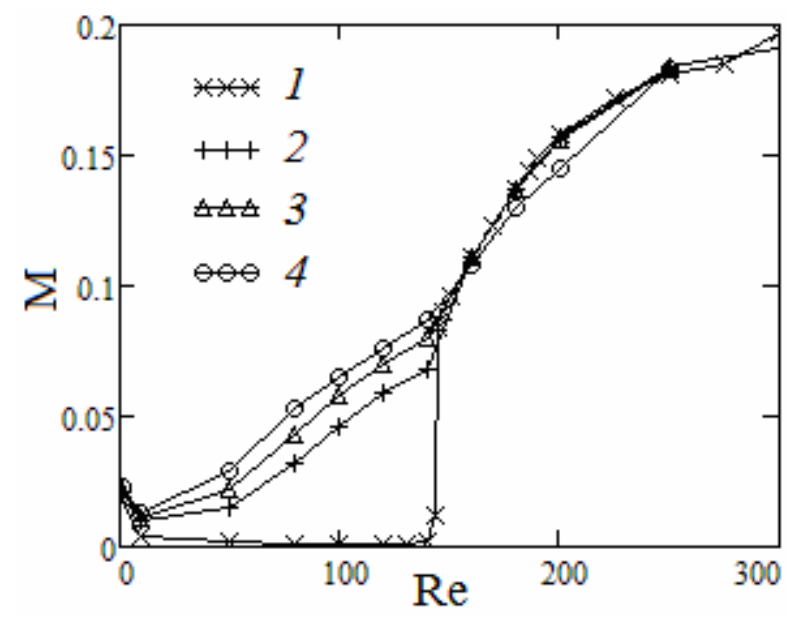

a)

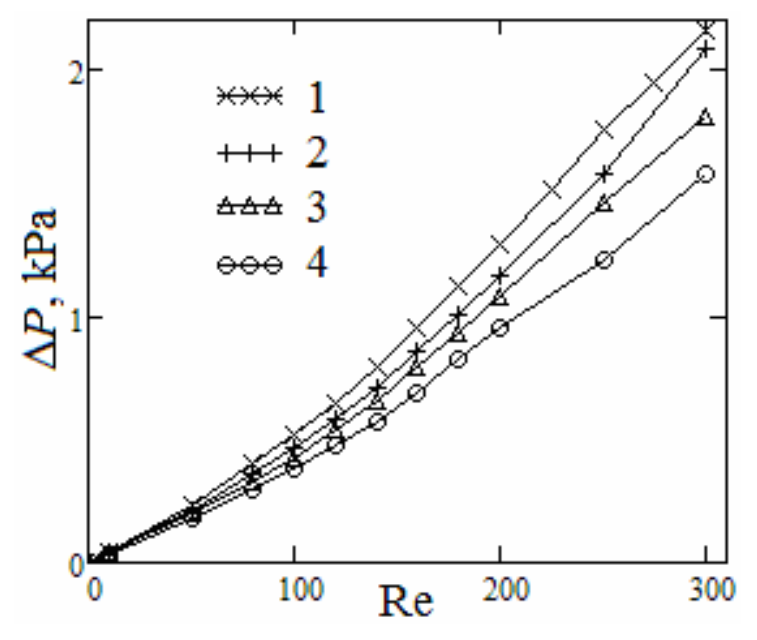

b)

Figure 2. Mixing efficiency (a) and pressure drop between the inlet and outlet of the mixing channel (b) versus Reynolds number, $\rho / \rho_{0}: 1$ (1), 1.25 (2), 1.5 (3), 2 (4)
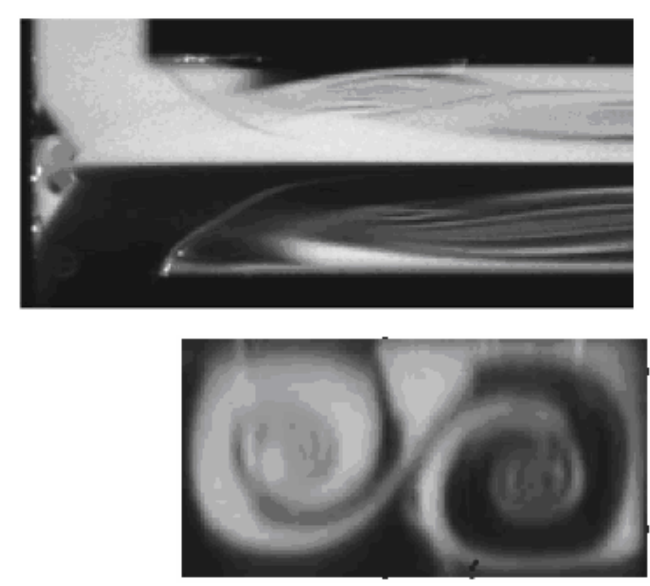

a)
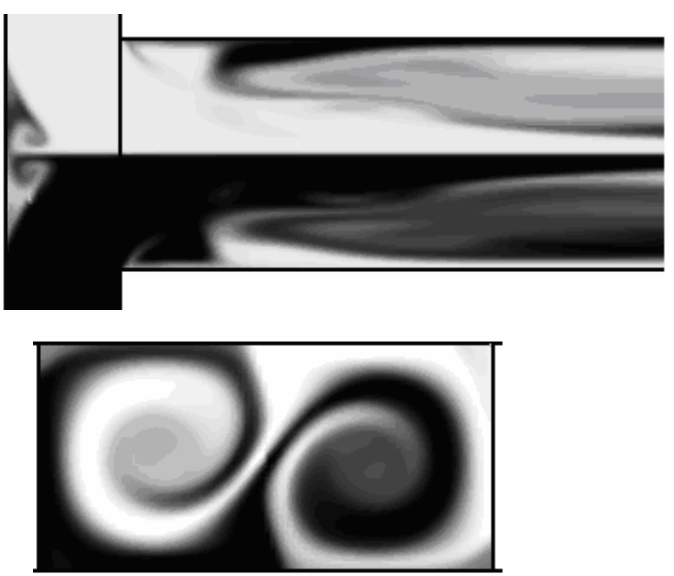

b)

Figure 3. Dye concentration contours at $\mathrm{Re}=186$ : Experiment [10] (a); numerical results (b)

Also an interesting behaviour of horseshoe vortices (Dean vortices) was observed (see Fig. 5). The Dean vortices structure was shown through $\lambda_{2}$ isosurfaces that is the second eigenvalue of the tensor $\left(\mathbf{S}^{2}+\Omega^{2}\right)$, where $\mathbf{S}$ is the strain rate tensor and $\Omega$ is the vorticity tensor. As is obvious, these vortices are generally preserved when the density of one of the fluids increased, though the increase in densities ratio leads to appearance of asymmetry relative to the central axis of the channel. As seen from Fig. 5, horseshoe vortex in the mixing channel 
grows faster in a fluid with a lower density. And the larger the densities ratio the more this process pronounced. Similar asymmetry is observed in the region of secondary vortices formation at the junction of the inlet channels and mixing channel. At that, one can see that the Dean horseshoe vortex, which is located in the more dense fluid, changes significantly with increasing the density of this fluid, in contrast to the vortex located in the water. With further increase in the Reynolds number flow reversal and the beginning of engulfment regime, similar to the flow of fluids with the identical parameters, is observed. In the course of numerical simulations analysis was revealed that this transition occurs within the region of Reynolds numbers from 144 to 144.5 , regardless of the densities ratio at the inlet of the Tshaped channel. Flow reversal and the transition from symmetric to engulfment flow regime are accompanied by a sharp increase in the mixing efficiency.

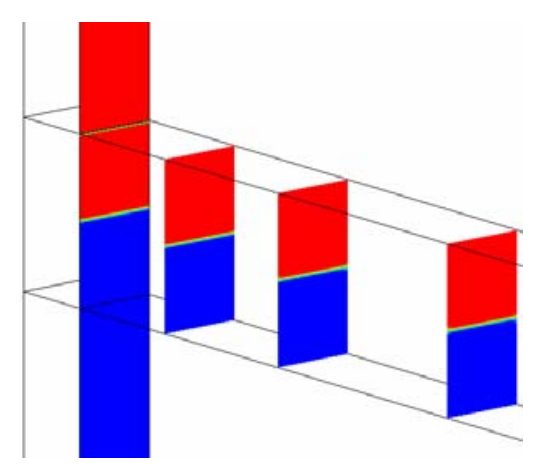

a)

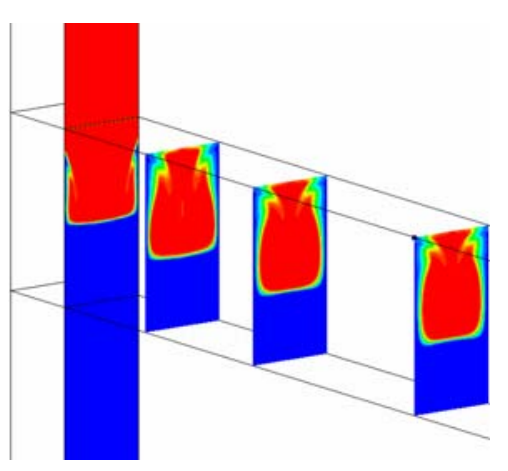

b)

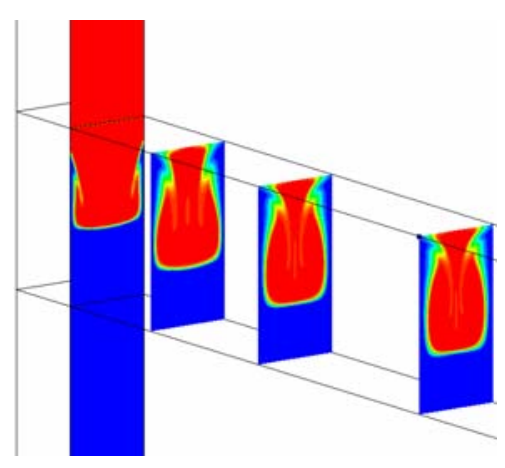

c)

Figure 4. Dye concentration contours at $\operatorname{Re}=120, \rho / \rho_{0}: 1$ (a); 1.5 (b); 2 (c)

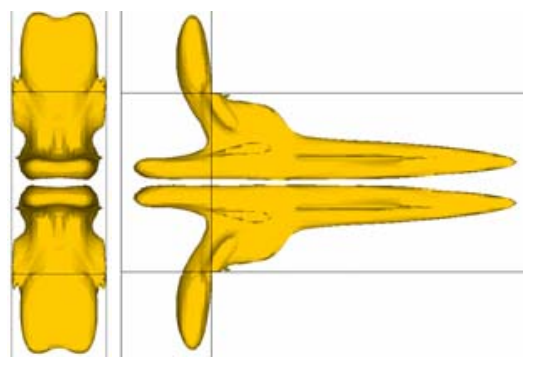

a)

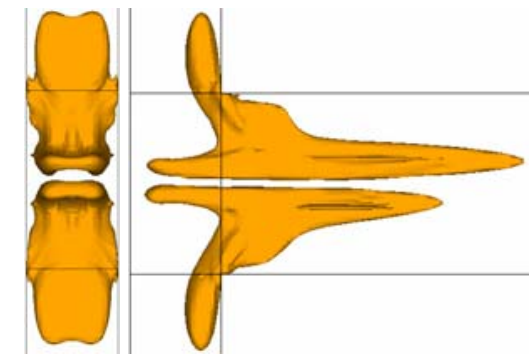

b)

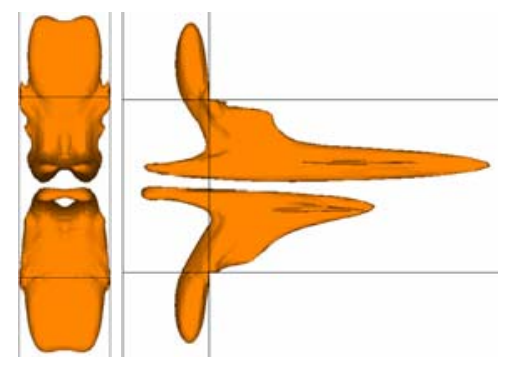

c)

Figure 5. $\lambda_{2}$ isosurfaces at $\operatorname{Re}=120, \rho / \rho_{0}: 1$ (a); $1.5(\mathrm{~b}) ; 2$ (c). The left part corresponds to mixer end-view; right part corresponds to side-view

In addition, it is seen that the mixing efficiency jump in the transition region becomes almost not pronounced up to its complete nonoccurrence, as it is observed for mixing of fluids with densities ratio equal to two (Fig. 2a). Mixing patterns in the engulfment regime are shown in Fig. 6. As is obvious, mixing structures at different densities ratios are qualitatively very similar, characterizing by similar formation of the layered S-shaped structure of miscible 
fluids. Miscible fluids interface in such layered structure is very well developed. It leads to a sharp increase in the mixing efficiency after flow reversal. However, some differences can be observed. The main difference is that the vortex in the denser fluid, which is formed in the mixing channel in the engulfment flow regime, develops much slower than similar vortex generated in the less dense one. This is well illustrated by the isosurfaces are shown in Fig. 7.

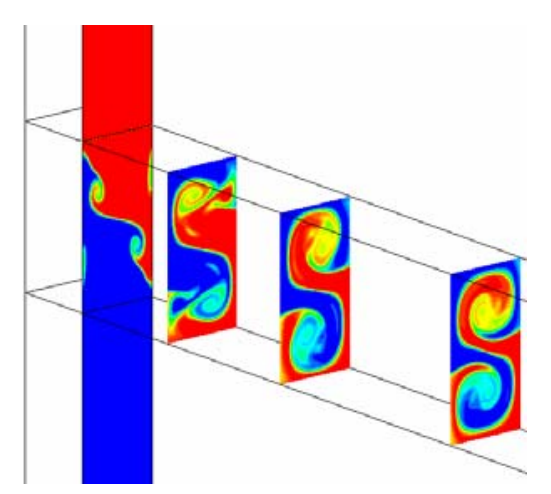

a)

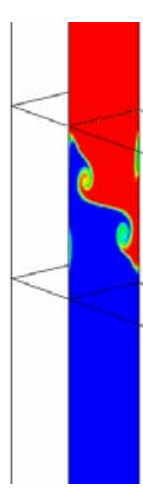

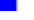

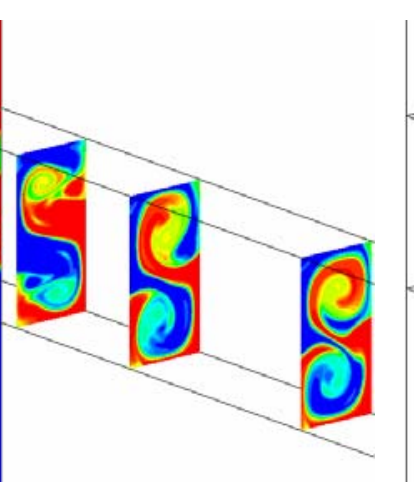

b)

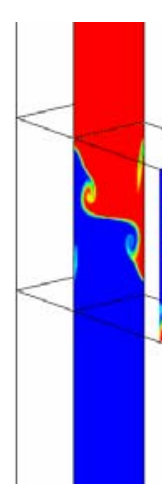

c)

Figure 6. Dye concentration contours at $\mathrm{Re}=250, \rho / \rho_{0}: 1$ (a); 1.5 (b); 2 (c)
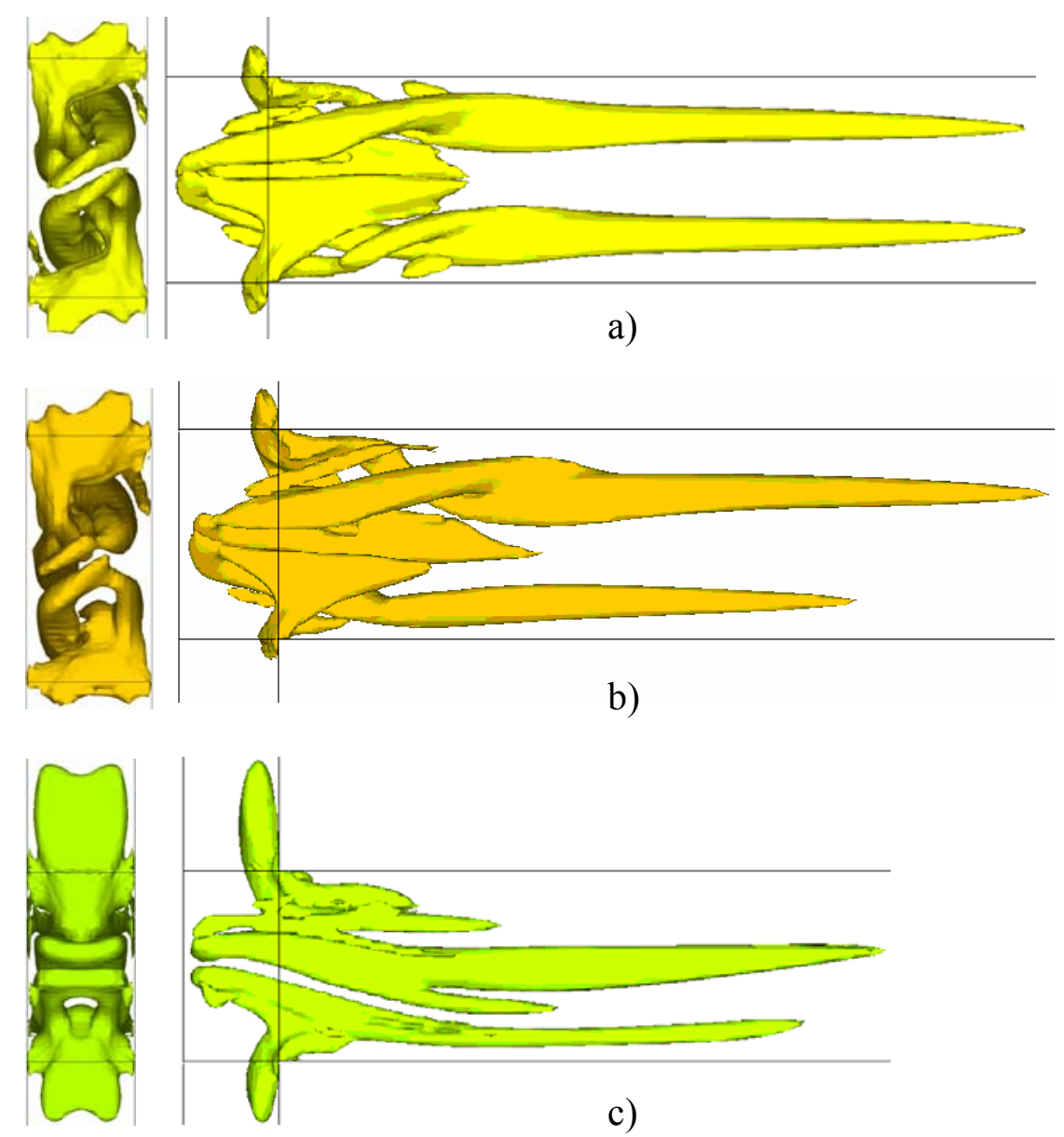

Figure 7. $\lambda_{2}$ isosurfaces at $\operatorname{Re}=250, \rho / \rho_{0}: 1$ (a); 1.5 (b); 2 (c). The left part corresponds to mixer end-view; right part corresponds to side-view 
As one can see, the vortex, located at the bottom of the mixing channel occupied by the denser fluid, is substantially shorter than the vortex located in the less dense fluid. With the increase of the densities ratio this difference is strengthened. The attenuation of one vortex in the mixing channel leads to the fact that the interface between mixing media in a denser fluid is not as developed as in the fluid with lower density. It is evidenced from a comparison in Fig. 6. In this regard, the mixing efficiency in the engulfment flow mode decreases with the increase in the densities ratio of mixing fluids (see Fig. 2a).

Also, the pressure drop between the inlet and outlet of the mixing channel was analyzed (see Fig. 2b). As is obvious, similarly to the case of $\rho / \rho_{0}=1$, the pressure drop is not characterized by any peculiarity in the transition region from the symmetric flow regime to asymmetric pattern. It is interesting that the pressure drop decreases with increasing densities ratio.

Effect of gravity in case of different fluids densities. Next, a series of calculations taking into account the non-zero gravity $\left(g=9.81 \mathrm{~m} / \mathrm{s}^{2}\right)$ was carried out. The micromixer was placed as shown in Fig. 1 and the gravity vector was directed down the mixer's height. The densities ratio of miscible fluids was equal to 2 . The mixer sizes were varied to better understanding the gravity influence on the flow and mixing patterns. The base configuration with height of $200 \mu \mathrm{m}$ is shown in Fig. 1. For other configurations the mixer's height was set equal to $100 \mu \mathrm{m}, 300 \mu \mathrm{m}$ and $400 \mu \mathrm{m}$. All another mixers sizes were obtained by multiplying the correspond dimension on the heights ratio. Calculations for the symmetric flow regime at $\mathrm{Re}=120$ and for the engulfment flow regime at $\mathrm{Re}=180$ were carried out. Dye concentration contours for these Reynolds numbers and different mixers configurations at a distance of 300 $\mu \mathrm{m}$ from the entrance of the mixing channel are shown in Fig. 8. All pictures in Fig. 8 scaled to uniform size for better understanding. The blue color corresponds to denser fluid. The dependence of the mixing efficiency on the mixer height for two different Reynolds numbers is shown in Fig. 9.

As can be seen from Fig. 8, the gravity has no affect on the shape of boundary between two media for the smallest sizes micromixer (compare Figs. 8a with Figs. 4c,6c); a slight deformation of the shape of boundary was observed for base configuration with height of $200 \mu \mathrm{m}$ (Figs. 8b); the significant affect of gravity on the shape of boundary between two media was observed for big micromixers $(h>200 \mu \mathrm{m})$. As one can see from Figs. 8c,d, the denser fluid moves down to the bottom wall of the mixing channel under the influence of gravity force in such configurations. At the same time, the less dense fluid is pushed out by the more dense one to the top wall of the mixing channel. Consequently, the presence of 
gravity significantly affects the mixing patterns for big micromixers and causes an increase of the interface between miscible media that leads to an increase of the mixing efficiency. But the discrepancy in mixing efficiency between zero gravity and non-zero gravity cases does not exceed $2.4 \%$ for the symmetric flow regime and $1.4 \%$ for the engulfment flow regime in the base configuration case ( $h=200 \mu \mathrm{m}$, see Fig. 9). Based on this, the effect of gravity for big micromixers with height more than $300 \mu \mathrm{m}$ should be taken into account. In turn, for the base configuration and smaller mixers $(h<200 \mu \mathrm{m})$ the effect of gravity can be neglected. Moreover, the residence time is not the same for different configurations, and the ratio of this quantity for bigger and smaller mixers is square-proportional to the ratio of their geometric dimensions. For bigger mixers, this time will be enough to achieve the stratification of the streams. It gives another reason for taking into account the effect of gravity for big micromixers with height more than $300 \mu \mathrm{m}$.

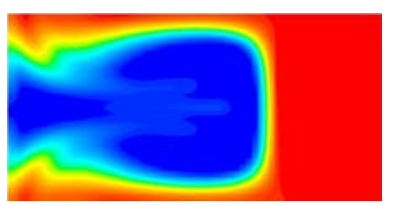

a)

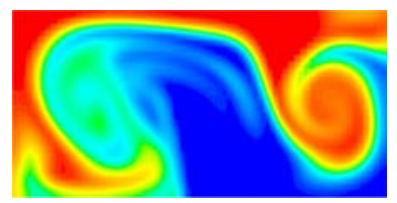

a)

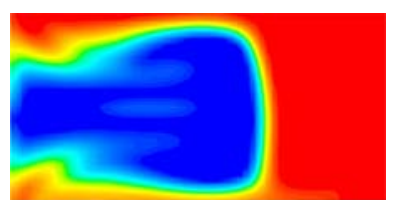

b)

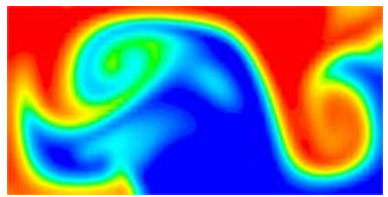

b)

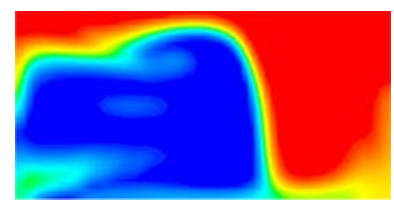

c)

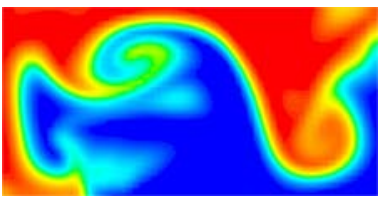

c)

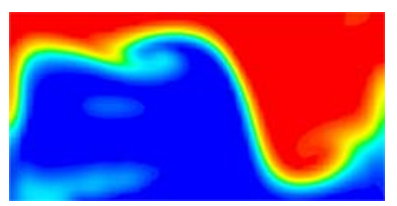

d)

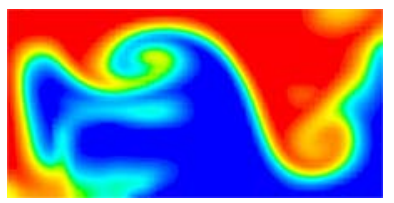

d)

Figure 8. Dye concentration contours at $\mathrm{Re}=120$ (upper) and $\mathrm{Re}=180$ (lower) for different sizes of micromixers $h: 100 \mu \mathrm{m}$ (a); $200 \mu \mathrm{m}$ (b); $300 \mu \mathrm{m}$ (c); $400 \mu \mathrm{m}$ (d)

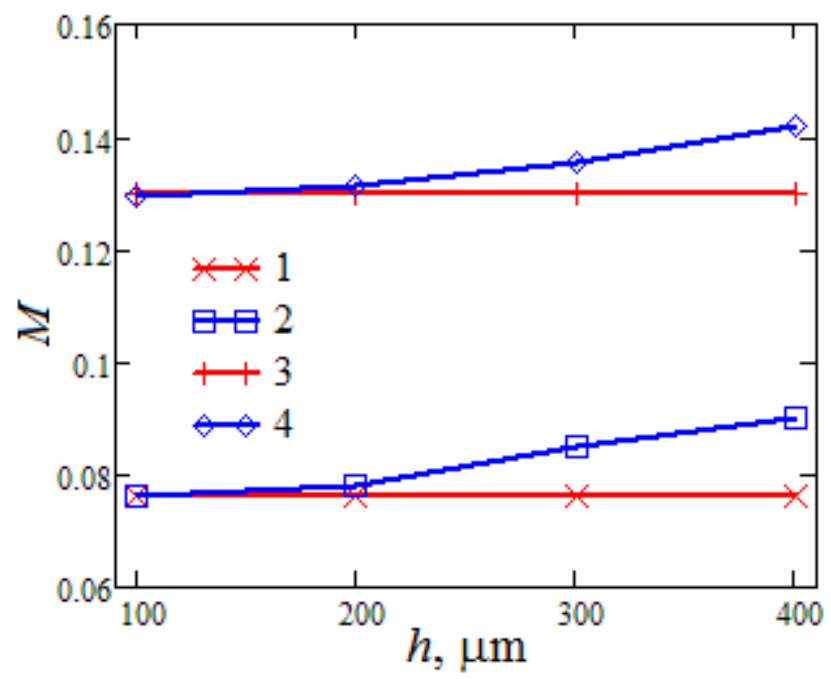

Figure 9. Mixing efficiency versus mixer size at $\mathrm{Re}=180$ (upper) and $\mathrm{Re}=120$ (lower): without gravity (curves 1 and 3); with gravity (curves 2 and 4) 
Effect of viscosity. Next, viscosity effect on flow regimes in studied micromixer was investigated. This series of calculations differed from the previous one by the fact that the viscosity of one of the mixing fluids was varied. As before, water tinted with rhodamine was supplied through one of the mixer inlets. Fluid with the same density as of water but with modified viscosity was supplied into the mixer through another inlet. Three series of calculations with fluid viscosities of $1.25,1.5$ and 2 times greater than the viscosity of water were carried out. The viscosity of water at room temperature $\mu=0.001 \mathrm{~Pa} \cdot \mathrm{s}$ was used to determine the Reynolds number.

The flow significantly changes when increasing the viscosity of one of the miscible fluids. For very small $(<10)$ Reynolds numbers the flow and mixing structure of fluids with different viscosities is generally similar the flow of two identical fluids. Therefore, the mixing efficiency in this regime weakly depends on the viscosity of the fluid (see Fig. 10a). The increase in Reynolds number leads to formation of vortices in the channel, and the difference in viscosities begins to affect. It is obvious, that in contrast to the case of equal viscosities of miscible fluids, the interface between media ceases to be flat. More viscous fluid pushes less viscous fluid from the channel walls, enveloping it. With the increase in viscosities ratio this effect is enhanced and the contact area of mixing media increases. This leads to enhancement of mixing efficiency with increase in the viscosities ratio in this regime (Fig. 10a).

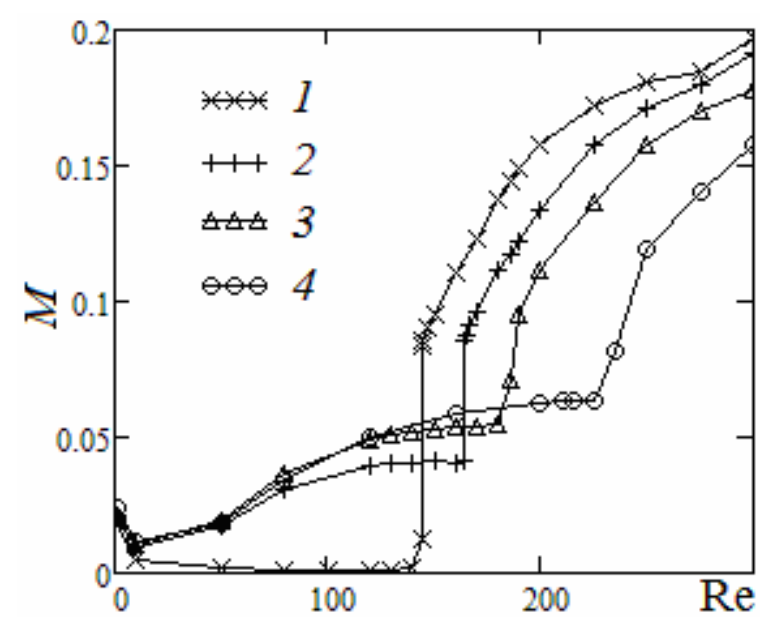

a)

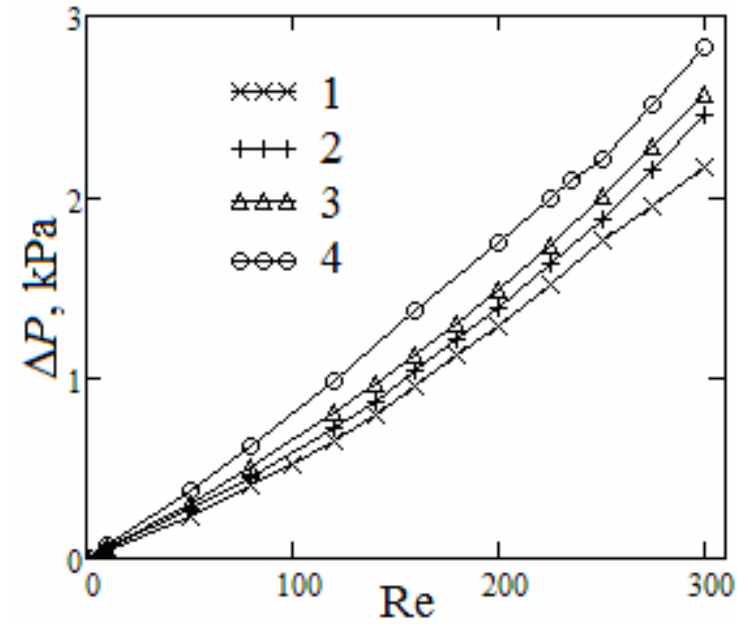

b)

Figure 10. Mixing efficiency (a) and pressure drop between the inlet and outlet of the mixing channel (b) versus Reynolds number, $\mu / \mu_{0}: 1$ (1); 1.25 (2); 1.5 (3); 2 (4)

Also, an interesting behavior of Dean horseshoe vortices is observed. As is obvious, these vortices are generally preserved when the viscosity of one of the fluids increased, though the increase in viscosities ratio leads to appearance of asymmetry relative to the 
central axis of the channel. The horseshoe vortex in the mixing channel attenuates faster in the fluid with the greater viscosity. At that, the higher the viscosities ratio, the more pronounced is this process. Similar asymmetry is observed in the region of secondary vortices formation at the junction of the inlet channels and mixing channel. At that, the Dean horseshoe vortex, which is located in water, practically does not change with increasing viscosity of the second fluid. It is revealed that the further increase in the Reynolds number also leads to flow reversal and beginning the engulfment flow regime. However, in contrast to the case of identical viscosities, for which the transition occurs within Reynolds numbers ranged from 144 to 144.5, the Reynolds numbers corresponding to flow transition in this case are shifted significantly: for viscosities ratio equal to 1.25 the transition occurs at the Reynolds numbers ranged from 164 to 165 ; for viscosities ratio equal to 1.5 flow reversal occurs within the range of Reynolds numbers from 181 to 183 ; while for the viscosities ratio equal to 2, Reynolds numbers relevant to flow reversal correspond to the range 222-227. It is very well seen in Fig. 10a, that represented the diagram of mixing efficiency versus Reynolds number.

Reversal and transition from symmetric to engulfment flow regime is accompanied by a sharp increase in mixing efficiency. The diagram shows that Reynolds numbers corresponding to flow transition are shifted to the higher values with increase of the viscosities ratio. In addition, it is seen that in the transition region, the jump in mixing efficiency becomes less pronounced. As is obvious, mixing structures at different viscosities ratios are qualitatively very similar and characterized by formation of the layered S-shaped structure of miscible fluids. The boundary between miscible fluids in such layered structure is very well developed that leads to a sharp increase in the mixing efficiency in engulfment regime. However, there are some differences. The main difference is that the vortex formed in more viscous fluid in the mixing channel after flow reversal attenuates much faster than the similar vortex in the water. This is well illustrated by isosurfaces shown in Fig. 11.

As one can see, the vortex, located at the bottom of the mixing channel, occupied by a more viscous fluid, is substantially shorter than a vortex in water. This difference increases with the increase in the viscosities ratio. The attenuation of one vortex in the mixing channel leads to the fact that the interface of mixing media in a more viscous fluid is not as well developed as in the water.

In this regard, the mixing efficiency in the engulfment flow regime decreases with the increase in the viscosities ratio of mixing fluids (see Fig. 10a). Also, the pressure drop between the inlet and outlet of the mixing channel was analyzed (Fig. 10b) and no specific features (in the region of the transition from symmetric mode to engulfment flow) for the 
more viscous fluids was revealed. Naturally, the increase in viscosity causes the increase in pressure drop.
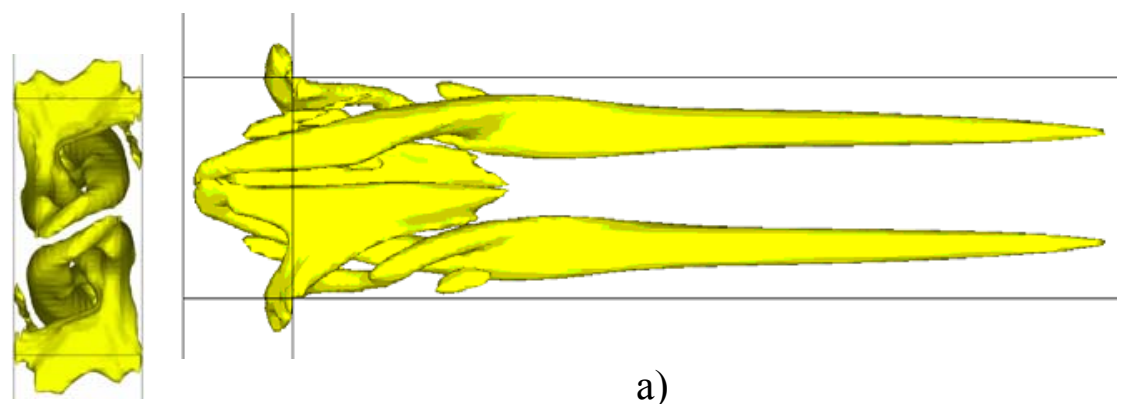

a)
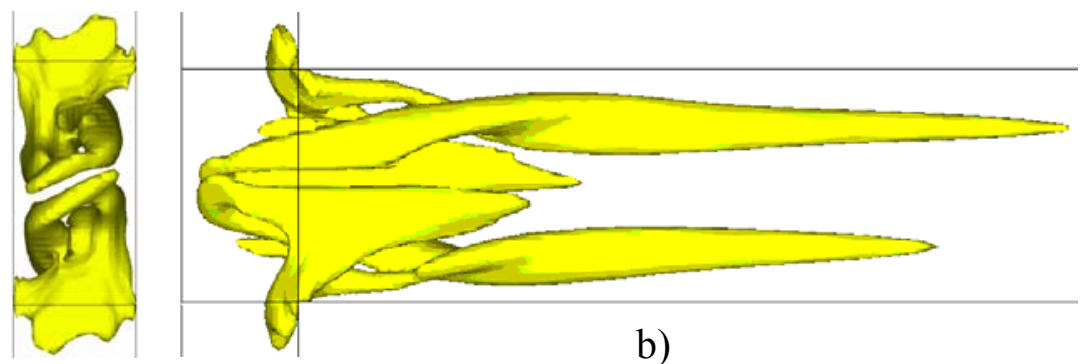

b)
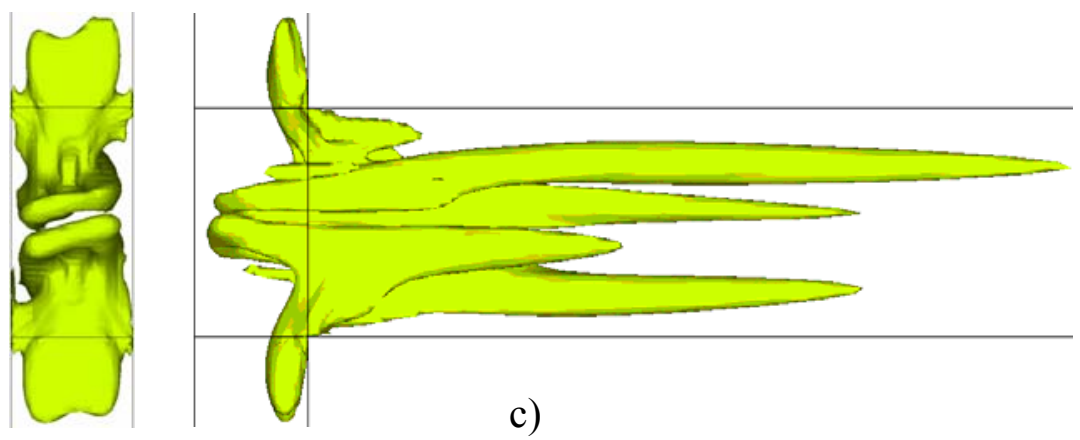

Figure 11. $\lambda_{2}$ isosurfaces at $\operatorname{Re}=250, \mu / \mu_{0}: 1$ (a); 1.5 (b); 2 (c). The left part corresponds to mixer end-view; right part corresponds to side-view

Effect of rheology. After that, the rheology effect on the flow regimes in micromixer was investigated. This series of calculations differed from the previous one by the fact that non-Newtonian fluid properties were set according to the formula $\mu(\dot{\gamma})=k \dot{\gamma}^{n-1}$. At that, $k=0.001$, while power coefficient $n$ varied and was equal to $0.3,0.5$ and 0.8 . All other parameters were similar to those in previous calculations.

The Reynolds number was varied in addition to the power coefficient. There is no common definition of the Reynolds number for non-Newtonian media, and thus various options can be used. In this work two options of defining the Reynolds number for nonNewtonian fluid was considered:

$$
\operatorname{Re}=\frac{\rho U^{2-n} d_{h}^{n}}{k} \quad \text { (a) } \quad \operatorname{Re}=\frac{\rho U^{2-n} d_{h}^{n}}{k \cdot 8^{n-1}\left(\frac{3 n+1}{4 n}\right)^{n}}
$$


where $\rho$ is the density, $U$ is the mean flow velocity in the mixing channel, $d_{h}$ is the equivalent diameter of the mixing channel.

Basically formula (4b) is used, and the majority of figures shown below correspond to calculations made by this formula. Figs. 12-17 show numerical results of non-Newtonian fluids mixing. The appearance of non-Newtonian properties of miscible fluids significantly changes the flow pattern. For very small Reynolds numbers the flow and mixing structure of fluids with different power coefficients $n$ is generally similar to the flow of two Newtonian fluids. The mixing efficiency in this regime is very small, however, with the increase in the power coefficient $n$ it reduces even more (Fig. 12a).

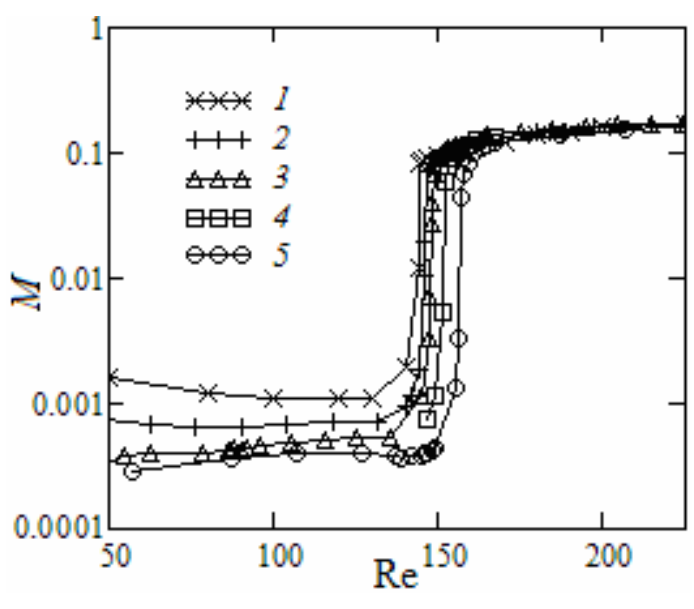

a)

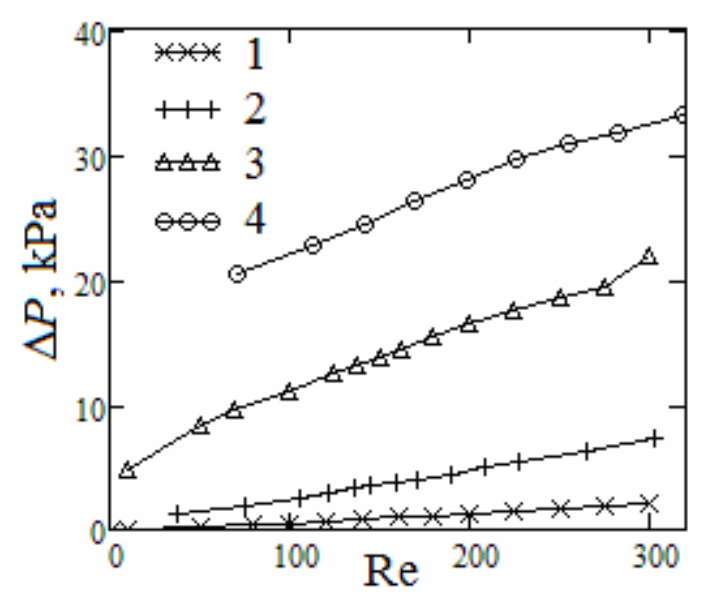

b)

Figure 12. Mixing efficiency (a), $n: 1$ (1); 0.8 (2); 0.5 (3); 0.4 (4); 0.3 (5); and pressure drop between the inlet and outlet of the mixing channel (b), $n$ : 1 (1); 0.8 (2); 0.5 (3); 0.3 (4) versus the Reynolds number

Despite this, qualitatively, these changes are almost imperceptible. This is well illustrated by Fig. 13. Though, there are certain differences. Approximately in the same region of Reynolds numbers as that for Newtonian fluids, the onset of engulfment regime, which is accompanied by a sharp increase in mixing efficiency, was observed in the mixing channel. However, with decrease of power coefficient $n$ this transition is shifted to the higher values of Reynolds numbers. This can be seen from Fig. 12a, that represented the mixing efficiency.

Also, an interesting behavior of Dean horseshoe vortices at different power coefficients $n$ is observed. It was revealed that these vortices generally persisted, though the central core of each of Dean vortices becomes ever more elongated with reduced power coefficient $n$. Similar process is observed in the region of secondary vortices formation at the junction of the input channels and mixing channel. It is seen that these secondary vortices are also strongly stretch and increase in size. Fig. 13 represents $\lambda_{2}$ isosurfaces at Reynolds 
number equal to 120 and different power coefficients $n$. Mixing patterns in the mixer in the engulfment regime at different power coefficients $n$ are qualitatively very similar (see Fig. 14). Miscible fluids form the layered S-shaped structure similarly to previous cases. The interface of miscible fluids in such layered structure is very well developed that leads to sharp increase in the mixing efficiency after the reversal. Interesting, that the change in the power coefficient $n$ practically does not affect the mixing of two non-Newtonian fluids in this region. It is well illustrated by the mixing efficiency shown in Fig. 12a. Fig. 14 shows $\lambda_{2}$ isosurfaces at Reynolds number equal to 180 and various values of power coefficient $n$. As is obvious, in general, the vortex structure of the flow is similar at various power coefficients $n$, except the length of vortices stretching along the mixing channel, that decreases with the increase of $n$. This is due to the fact that an increase of power coefficient $n$ results in increase of effective viscosity, and therefore, the vortices decay much faster.

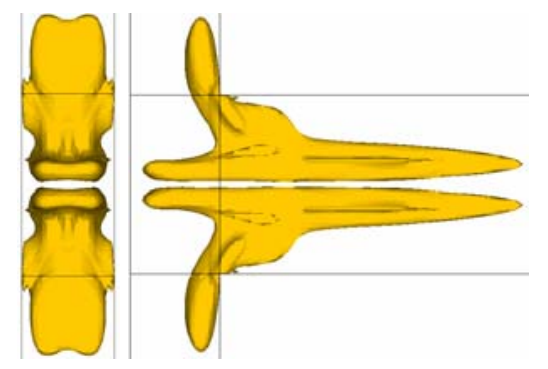

a)

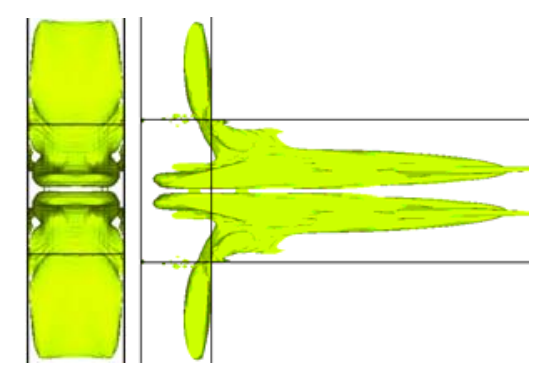

c)

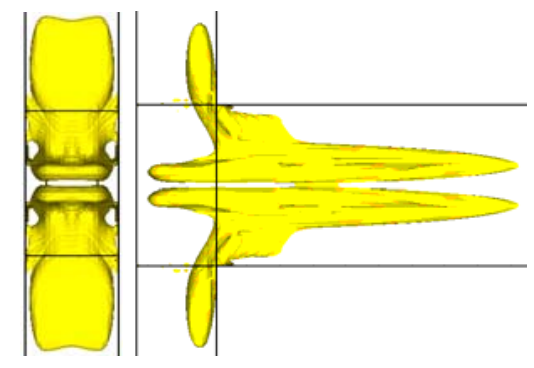

b)

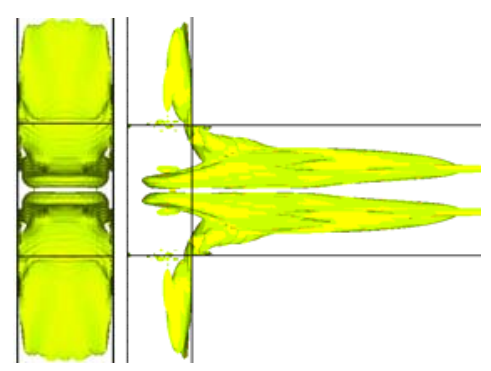

d)

Figure 13. $\lambda_{2}$ isosurfaces at $\mathrm{Re}=120$ and power coefficients $n: 1$ (a); 0.8 (b); 0.5 (b); 0.3 (c).

The left part corresponds to mixer end-view; right part corresponds to side-view

Also, the pressure drop between the inlet and outlet of the mixing channel was analyzed (Fig. 12b) and practically no difference was revealed (does not exceed a tenth of a percent). Naturally, the increase of $n$ causes the increase in pressure drop. The fact that with decreasing power coefficient $n$ the transition to the engulfment regime in the mixing channel is shifted to the higher values of Reynolds numbers is new and not previously noted in other studies. The shift of the transition region with increase of power degree $n$ is observed even more clearly in case of calculating the Reynolds number by the formula $4 \mathrm{a}$. 

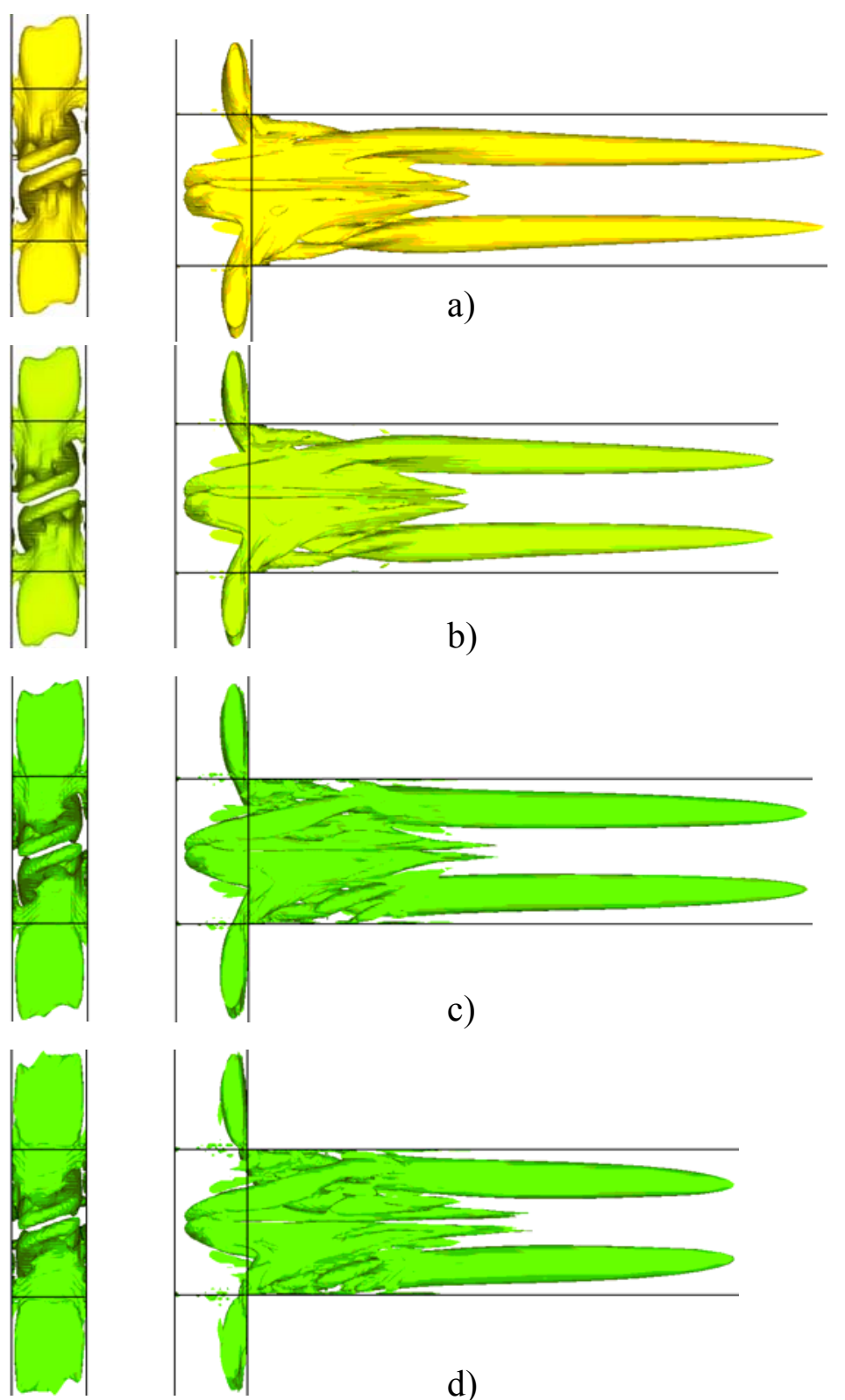

Figure 14. $\lambda_{2}$ isosurfaces at $\mathrm{Re}=180$ and power coefficients $n: 1$ (a); 0.8 (b); 0.5 (c); 0.3 (d).

The left part corresponds to mixer end-view; right part corresponds to side-view

Dependences of mixing efficiencies on the Reynolds number calculated by the formula 4a for different power coefficients $n$ are presented in Fig. 15a. It is obvious that the Reynolds number corresponding to the transition from one flow regime to another is changing from 145 to 600 when reducing the power coefficient $n$ from 1 to 0.3 . The critical Reynolds number, corresponds to a change of regimes, versus power coefficient $n$ is shown in Fig. 15b. As is obvious, it increases with the decreases of the power coefficient $n$, and this occurs according to the nonlinear law, close to the hyperbole. 


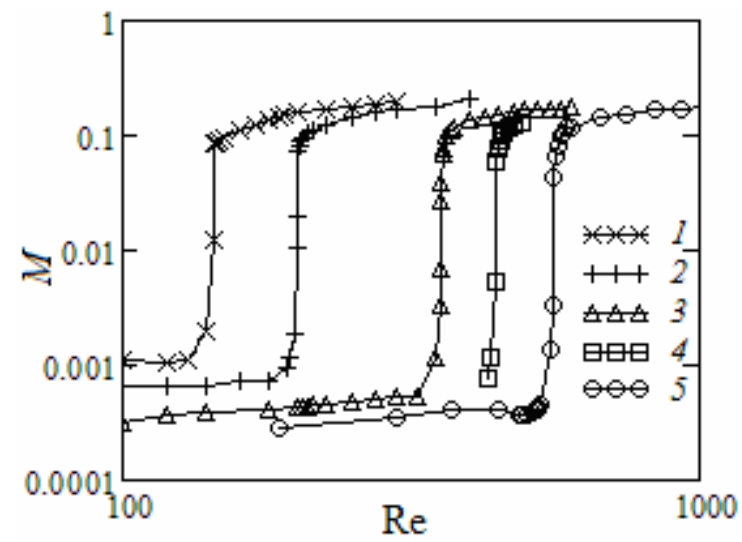

a)

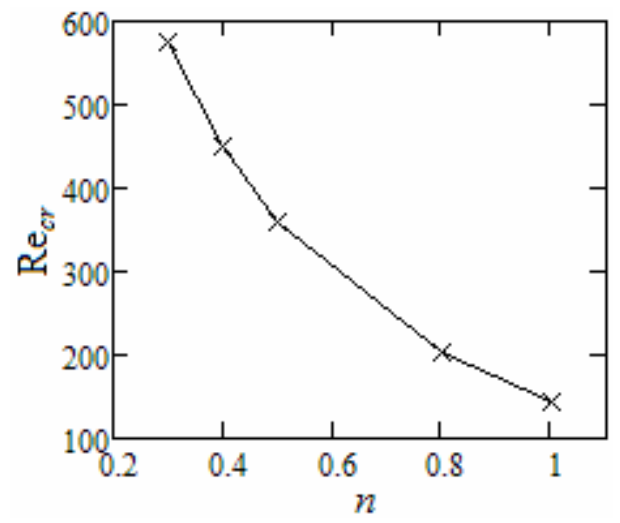

b)

Figure 15. Mixing efficiency versus Reynolds number (a), calculated by the formula (4a), $n: 1$ (1); 0.8 (2); 0.5 (3); 0.4 (4); 0.3 (5); critical Reynolds number versus $n$ (b).

Effect of the initial temperature. In addition, thermal mixing of Newtonian fluids in T-shaped micromixer was studied. The effect of different initial temperatures of the fluids at the inlets on the flow regimes was investigated. In this series of calculations the temperature of one of the fluids was set equal to $20^{\circ} \mathrm{C}$, while the temperature of other fluid varied and was equal to: $10^{\circ} \mathrm{C}, 30^{\circ} \mathrm{C}, 40^{\circ} \mathrm{C}$, and $50^{\circ} \mathrm{C}$. Pure water with the well-known temperaturedependent thermophysical properties [38-40] was used.

Fluids have different thermal properties at various fluid temperatures at the channel inlet. Mainly the mixing mode of two fluids in micromixer will be influenced by the fluid viscosity and density. As shown above, increasing the viscosity of one of the fluids shifts the region of transition from steady symmetrical vortex flow to the engulfment regime and increasing the density of one of the fluids causes the appearance of asymmetry in the flow of this fluid relative to the other. Both of these effects manifest themselves in the case of mixing of two fluids with different temperatures at the inlet to micromixer. The fluid with a greater viscosity begins gradually "flowing around" the fluid with a lower viscosity, as it was shown for the case of different viscosities. However, since densities of fluids are also different, therefore, with the increase in Reynolds number, the fluid with a higher density begins in turn "flowing around" the fluid with a lower density, as was shown above for the case of different densities. In addition, it was shown that the beginning of the engulfment regime depends only on the viscosities ratio and does not depend on the densities ratio. Further, with the increase in Reynolds number the engulfment flow regime begin and continue to develop. This is accompanied by increase of the vortices size, the interface area and, accordingly, the mixing efficiency.

Furthermore, as was shown above, the difference in the fluids densities may cause 
some changes in flow and mixing patterns due to the influence the natural convection in nonzero gravity case. There is the dimensionless number that expresses the ratio of the buoyancy term to the flow shear term and called the Richardson number (Ri). In thermal convection problems, Richardson number represents the importance of natural convection relative to the forced convection. The Richardson number in this context is defined as $\mathrm{Ri}=g \beta\left(T_{h}-T_{c}\right) L U^{-2}$, where $g$ is the gravitational acceleration, $\beta$ is the thermal expansion coefficient, $T_{h}$ is the hot fluid temperature, $T_{c}$ is the cold fluid temperature, $L$ is the characteristic length, and $U$ is the characteristic velocity. The Richardson number can also be expressed by using a combination of the Grashof number and Reynolds number, $\mathrm{Ri}=\mathrm{Gr} \mathrm{Re}^{-2}$. The Grashof number $(\mathrm{Gr})$ is a dimensionless number in fluid dynamics and heat transfer which approximates the ratio of the buoyancy to viscous force acting on a fluid. For pipes and channels the Grashof number is defined as $\mathrm{Gr}=g \beta\left(T_{h}-T_{c}\right) L^{3} v^{-2}$, where $v$ is the kinematic viscosity. Typically, the natural convection is negligible when $\mathrm{Ri}<0.1$, forced convection is negligible when $\mathrm{Ri}>10$, and neither is negligible when $0.1<\mathrm{Ri}<10$.

In our case the maximum temperature difference was $30^{\circ} \mathrm{C}\left(T_{h}=50^{\circ} \mathrm{C}, T_{c}=20^{\circ} \mathrm{C}\right)$. In these conditions the average thermal expansion coefficient $\beta_{a v}=3.155 \mathrm{~K}^{-1}$, the average kinematic viscosity $v_{a v}=0.781 \cdot \mathrm{mm}^{2} / \mathrm{s}$ and $L=h=200 \mu \mathrm{m}$. Eventually $\mathrm{Gr}=1.218$. The Reynolds number in the calculations was ranged from 10 to 300 , so the Richardson number was ranged from $1.353 \cdot 10^{-5}$ (for $\operatorname{Re}=300$ ) to 0.01218 (for $\operatorname{Re}=10$ ). Based on this, the natural convection can be neglected. This is also confirmed by the above calculations of the non-zero gravity effect, where for base configuration the maximum discrepancy in mixing efficiency between zero gravity and non-zero gravity cases is not exceed $2.4 \%$ for densities ratio $\rho / \rho_{0}=2$. Taking into the well-known temperature-dependent thermophysical properties of pure water [38-40], it can be found that the water density at $20^{\circ} \mathrm{C}$ is $\rho_{20}=998.2 \mathrm{~kg} / \mathrm{m}^{3}$, water density at $50^{\circ} \mathrm{C}$ is $\rho_{50}=988.1 \mathrm{~kg} / \mathrm{m}^{3}$, and the densities ratio is $\rho_{20} / \rho_{50}=1.01$. So, the gravity effect is negligible here.

The distribution of temperature and concentration of mixing components in various sections of the mixer for $\Delta t=10^{\circ} \mathrm{C}$ and different Reynolds numbers is shown in Fig. 16. As is obvious, the distributions of concentration and temperature are not absolutely identical. The thickness of the mixing layer of temperature field is considerably higher than that of the concentration field. This is due to the different scales of the heat conduction and diffusion processes. Thus, the case of variable-temperature fluid cannot be considered absolutely identical to the cases of variable viscosity and density of mixing media. 


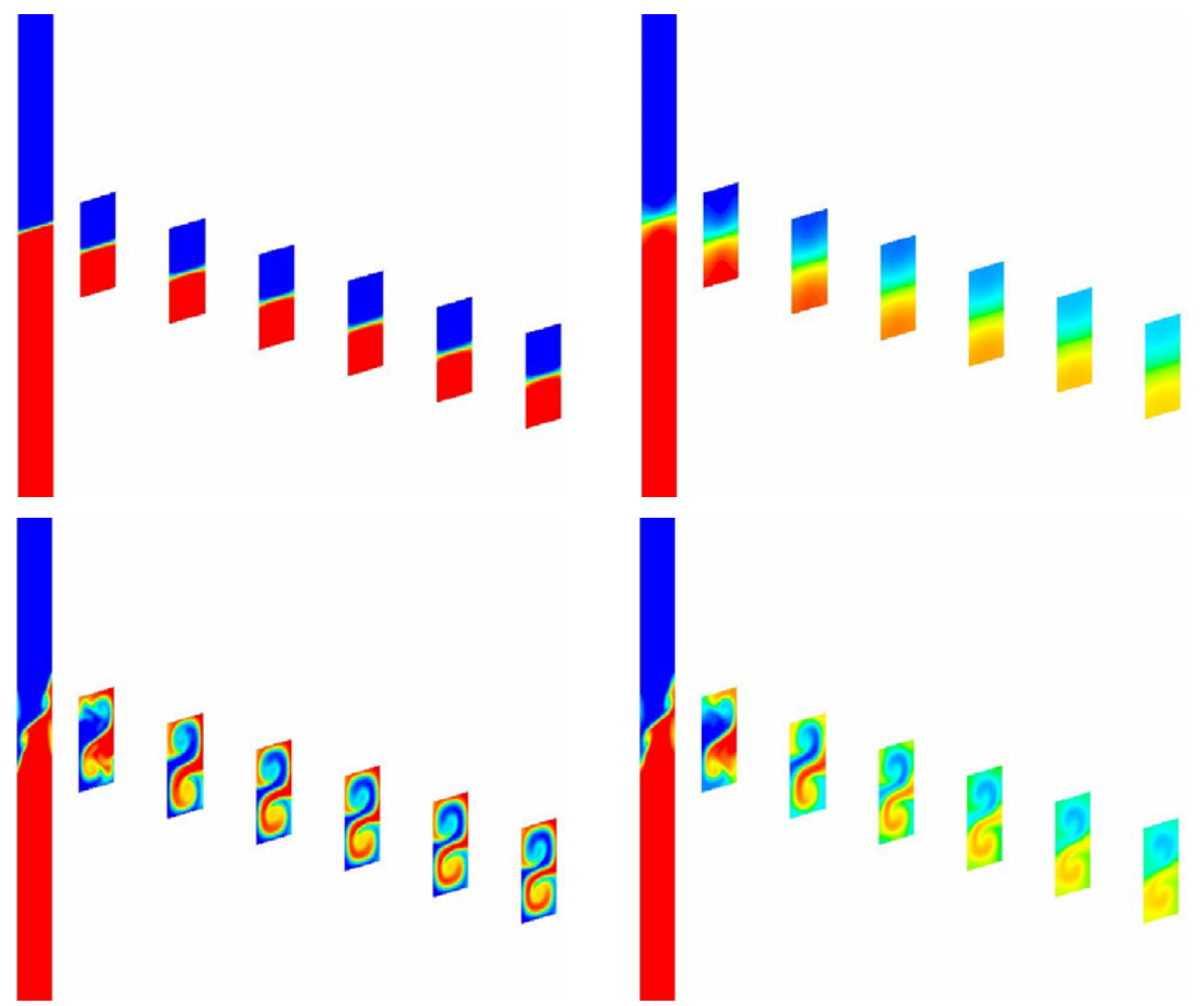

Figure 16. Concentration (left) and temperature (right) distribution at $\mathrm{Re}=10$ (upper) and

$$
\operatorname{Re}=150 \text { (lower) }
$$

Fig. 17a represented mixing efficiencies depending on the Reynolds number for different initial temperatures of the fluids. Analysis of the calculation results shows that change of flow regime at the Reynolds number equal to 162 occurs at a temperature difference equal to $-10^{\circ} \mathrm{C}$, while at $\operatorname{Re}=126$ for $\Delta t=10^{\circ} \mathrm{C}$, at $\operatorname{Re}=118$ for $\Delta t=20^{\circ} \mathrm{C}$, and at $\operatorname{Re}=114$ for $\Delta t=30^{\circ} \mathrm{C}$. Certainly, this is largely due to the viscosity effect. An interesting effect can be obtained if the reduced Reynolds number is calculated using the arithmetic mean value of $\mu_{m}=0.5 \cdot\left(\mu_{0}+\mu_{1}\right)$, where $\mu_{0}$ is the viscosity of water at $20^{\circ} \mathrm{C}$, while $\mu_{1}$ is the viscosity of water at some other temperature. Different ways of defining the effective viscosity and their influence on the obtained results were considered in [18]. Using this method of defining the reduced Reynolds number, it is possible to plot a dependence of two fluids mixing efficiency on this number (see Fig. 17b). It is obvious that the transition occurs at reduced Reynolds number equal or close to 145 and the mixing efficiency is approximately the same for all studied cases. Besides, the pressure drop between the inlet and outlet of the mixing channel was analyzed and its dependence on Reynolds number is shown in Fig. 18. It is apparent that these curves are arranged in order of increasing the initial inlet temperature of the fluid. This behavior is easy to explain, since the pressure drop is proportional to the fluid 
viscosity and density, therefore the increase of the inlet fluid temperature leads to decrease in pressure drop.

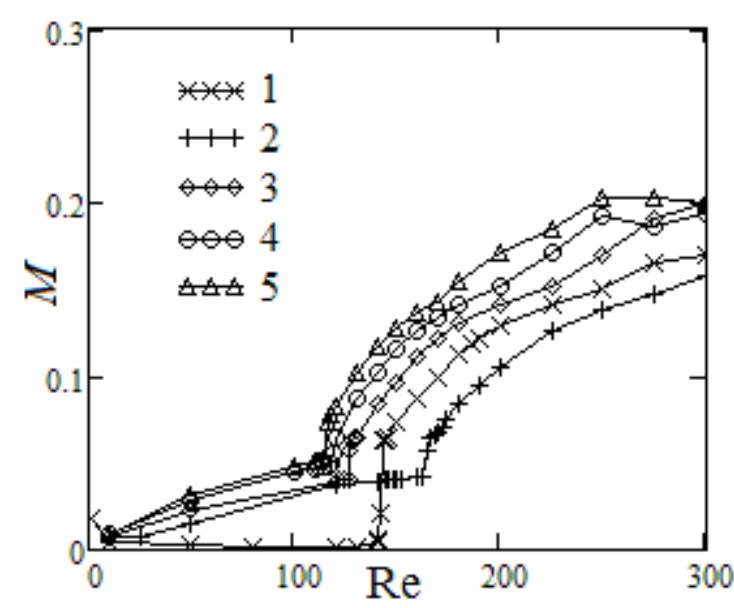

a)

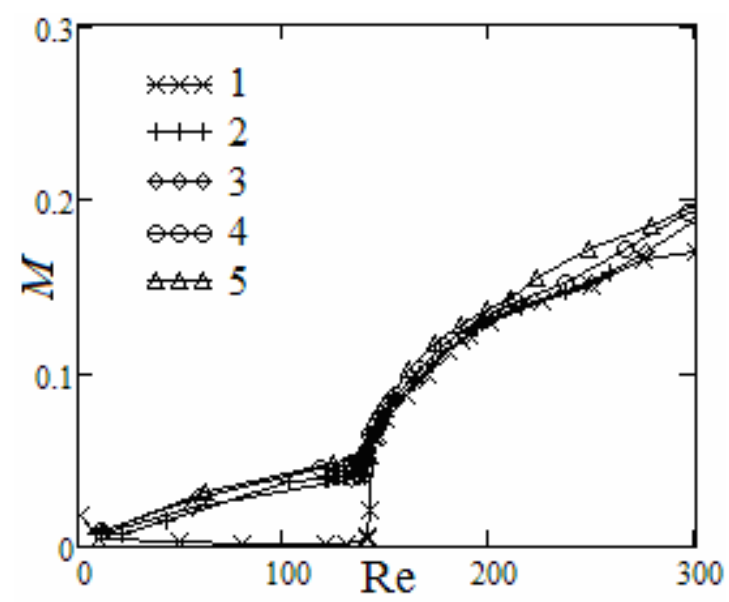

b)

Figure 17. Mixing efficiency versus Reynolds number and reduced Reynolds number, $\Delta t: 0^{\circ} \mathrm{C}(1) ;-10^{\circ} \mathrm{C}(2) ; 10^{\circ} \mathrm{C}(3) ; 20^{\circ} \mathrm{C}(4) ; 30^{\circ} \mathrm{C} \mathrm{(5).}$

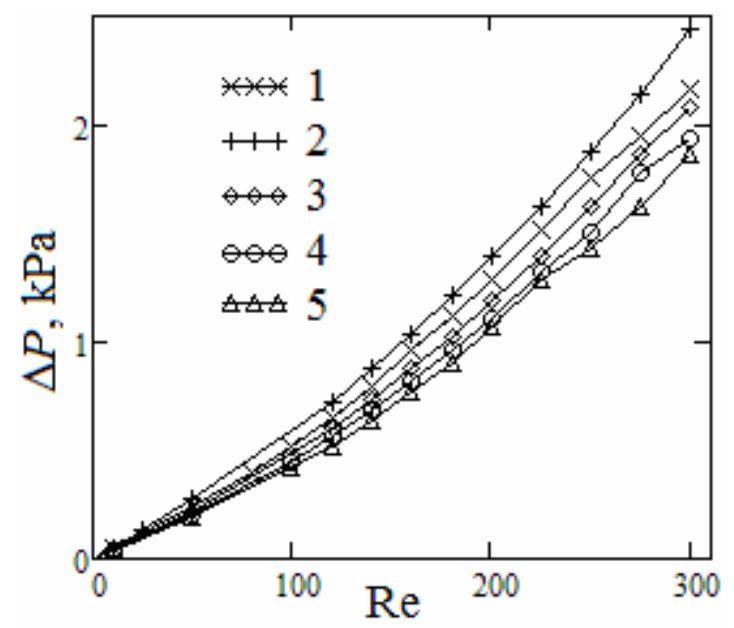

Figure 18. Pressure drop between the inlet and outlet of the mixing channel versus Reynolds number, $\Delta t: 0^{\circ} \mathrm{C}(1) ;-10^{\circ} \mathrm{C}(2) ; 10^{\circ} \mathrm{C}(3) ; 20^{\circ} \mathrm{C}(4) ; 30^{\circ} \mathrm{C}(5)$.

\section{CONCLUSION}

The effects of various parameters on the mixing efficiency of fluids were studied numerically, as well as fluids flow regimes and mixing modes. The conducted numerical simulation allowed identifying following regimes for incompressible fluid flow in T-shaped micromixer: stationary vortex-free flow; stationary symmetric vortex flow; intermittent transition from symmetric to asymmetric flow (engulfment flow); and stationary asymmetric vortex flow.

It was shown that the change in the density of one of the miscible fluids does not lead 
to a shift of flow regimes. However, a significant flow asymmetry in the region before its transition to the engulfment flow mode was observed. It was established that the mixing efficiency in the symmetric flow region increases with the increase in densities ratio. This is caused by an increase of the interface between miscible media because of the flow of more dense fluid around the less dense fluid. Besides, it was established that the effect of gravity for big micromixers with height more than $300 \mu \mathrm{m}$ should be taken into account. In turn, for the base configuration and smaller mixers $(h<200 \mu \mathrm{m}))$ the effect of gravity can be neglected. The maximum discrepancy in mixing efficiency between zero gravity and non-zero gravity cases does not exceed $2.4 \%$ for base configuration.

It was shown that the change in viscosity of one of the miscible fluids leads to a shift of flow regimes. However, in general, the above described flow regimes are preserved. It was revealed that the efficiency of mixing in the symmetric flow region increases with increase in the viscosities ratio due to the increase in the interface area of miscible media caused by flowpast of the more viscous fluid over the less viscous one. The mixing efficiency in asymmetric regime decreases with the increase in the viscosities ratio due to the more rapid attenuation of the S-shaped vortex structure in a more viscous fluid and a decrease in the interface area of miscible media. It was found that the flow of two fluids with different viscosities can be considered as self-similar relative to the reduced Reynolds number.

It was shown that the mixing of two non-Newtonian media in the T-shaped micromixer, similarly as for Newtonian fluids, is characterized by a transition from symmetric to asymmetric flow regime. The effect of power coefficient $n$ on the flow structure before and especially after the transition to engulfment flow regime that leads in particular to a shift of the flow regimes in considered micromixer was determined. This transition to the engulfment flow regime occurs at higher Reynolds numbers with increasing the power coefficient $n$. At that, the mixing efficiency in the symmetric flow regime decreases with decreasing of power coefficient $n$, while in the asymmetric flow regime is remains practically independent of $n$.

It was shown that the presence of a difference in initial temperatures of miscible fluids also leads to a shift of flow regimes. The simultaneous effect of differences in the viscosities and densities due to different initial temperatures of the fluids on the mixing efficiency was revealed. It was also shown that the flow and mixing of two fluids with different initial temperatures can be considered as self-similar pattern with regard to the reduced Reynolds number. Besides, the considerable effect of the difference in initial temperatures of miscible fluids on a pressure drop between the outlet and inlet of micromixer with variable temperature water was revealed. It was also shown that the natural convection can be neglected. 
The work is performed at partial financial support of the Ministry of Education and Science of the Russian Federation within the state assignment with Siberian Federal University in 2017 (No. 16.8368.2017). The results of the investigation of temperature affect was funded by Russian Foundation for Basic Research, Government of Krasnoyarsk Territory, Krasnoyarsk Region Science and Technology Support Fund (Contract No. 16-48243061). The results of the investigation of rheology affect were obtained within the Russian Science Foundation grant (project No. 17-79-20218).

\section{REFERENCES}

1. Tabeling $P$. Introduction to microfluidics. Oxford: Oxford University Press, 2005

2. Karnidakis G., Beskok A., Aluru N. Microflows and nanoflows // Interdisciplinary Applied Math. Springer Science+Business Media Inc., 2005. V. 29. 817 p.

3. Karnik R. Microfluidic mixing // Encyclopedia of microfluidics and nanofluidics / Ed. Li D. Springer, 2008. P. 1177

4. Rudyak V., Minakov A. Modeling and optimization of Y-type micro-mixers // Micromachines, 2014. Vol. 5. Is. 4. Pp. 886-912

5. Aubin J., Fletcher D.F., Xuereb C. Design of micro-mixers using CFD modeling // Chem. Eng. Sci., 2005. Vol. 60. Iss. 8-9, Pp. 2503-2516

6. Mansur E., Mingxing Y., Yundong W., Youyuan D. A state-of-the-art review of mixing in microfluidic mixers // Chinese J. Chem. Eng. 2008. Vol. 16. Is. 4. Pp. 503-516

7. Minakov A.V., Rudyak V.Ya., Gavrilov A.A., Dekterev A.A. On optimization of mixing process of fluids in microchannels // J. of Siberian Federal University. Math. \& Phys., 2010. Vol. 3. Is. 2. Pp. 146-156

8. Rudyak V.Ya., Minakov A.V., Gavrilov A.A., Dekterev A.A. Modelling of flows in micromixers // Thermophysics and Aeromechanics, 2010. Vol. 17. Is. 4. Pp. 565-576

9. Bokenkamp D., Desai A., Yang X., Ta, Y. C., Marzluff E.M., Mayo S. L. Microfabricated silicon mixers for submillisecond quench-flow analysis // Anal. Chem., 1998, Vol. 70. Is. 2. Pp. 232-236

10. Hoffmann M., Schluter M., Rubiger N. Experimental investigation of fluid-fluid mixing in T-shaped micro-mixers using $\mu$-LIF and $\mu$-PIV // Chem. Eng. Sci., 2006. Vol. 61. Is. 9. Pp. 2968-2976

11. Bothe D., Stemich C., Warnecke H.-J. Theoretische und Experimentelle Untersuchungen der Mischvorgänge in T-förmigen Mikroreaktoren - Teil 1: Numerische Simulation und Beurteilung des Strömungsmischens // CIT, 2006. Vol. 76. Is. 10. Pp. 1480-1484 
12. Bothe D., Stemich C., Warnecke H.-J. Fluid mixing in a T-shaped micro-mixer // Chem. Eng. Sci., 2006. V. 61. Is. 9. Pp. 2950-2958

13. Stemich $C$. Theoretische und numerische Untersuchung des Struomungsmischens in einem T-formigen Mikromischer. Dissertation zur Erlangung des akademischen Grades Doktor der Naturwissenschaften. Universit at Paderborn, 2006.

14. Dreher S., Kockmann N., Woias P. Characterization of laminar transient flow regimes and mixing in T-shaped micro-mixers // Heat Trans. Eng., 2009. Vol. 30. Is. 1-2. Pp. 91-100

15. Minakov A.V., Rudyak V. Ya., Gavrilov A. A., Dekterev A.A. Mixing in T-shaped micromixer at moderate Reynolds numbers // Thermophysics and Aeromechanics, 2012. Vol. 19. Is. 3. Pp. 385-395

16. Minakov A., Rudyak V., Dekterev A., Gavrilov A. Investigation of slip boundary conditions in the T-shaped microchannel // Int. J. of Heat and Fluid Flow, 2013. Vol. 43. Pp. 161-169

17. Minakov A., Yagodnitsina A., Lobasov A., Rudyak V., Bilsky A. Study of fluid flow in micromixer with symmetrical and asymmetrical inlet conditions // La Houille Blanche, 2013. Is. 5. Pp. 12-21

18. Lobasov A.S., Minakov A.V., Rudyak V.Ya. Viscosity effect on the flow modes in T-type micro-mixers // Fluid Dynamics, 2016. Vol. 51. Is. 3. Pp. 381-388

19. Galletti C., Arcolini G., Brunazzi E., Mauri R. Mixing of binary fluids with compositiondependent viscosity in a T-shaped micro-device // Chem. Eng. Sci., 2015. Vol. 123. Pp. 300-310

20. Galletti C., Brunazzi E., Mauri R. Unsteady mixing of binary liquid mixtures with composition-dependent viscosity // Chem. Eng. Sci., 2017. Vol. 164. Pp. 333-343

21. Orsi G., Roudgar M., Brunazzi E., Galletti C, Mauri R. Water-ethanol mixing in T-shaped microdevices // Chem. Eng. Sci., 2013. Vol. 95. Pp. 174-183

22. Wang W., Zhao S., Shao T., Jin Y., Cheng Y. Visualization of micro-scale mixing in miscible liquids using $\mu$-LIF technique and drug nano-particle preparation in T-shaped micro-channels // Chem. Eng. J.. 2012. Vol. 192. Pp. 252-261

23. Afzal A., Kim K.-Y. Flow and mixing analysis of non-Newtonian fluids in straight and serpentine microchannels // Chem. Eng. Sci., 2014. Vol. 116. P. 263-274

24. Poole R.J., Alfateh M., Gauntlett A.P. Bifurcation in a T-channel junction: Effects of aspect ratio and shear-thinning // Chem. Eng. Sci., 2013. Vol. 104. Pp. 839-848

25. Andreussi T., Galletti C., Mauri R., Camarri S., Salvetti M.V. Flow regimes in T-shaped micro-mixers // Comp. and Chem. Eng., 2015. Vol. 76. Pp. 150-159 
26. Soleymani A., Yousefi H., Turunen I. Dimensionless number for identification of flow patterns inside a T-micromixer // Chem. Eng. Sci., 2008. Vol. 63. Pp. 5291-5297

27. Fani A., Camarri S., Salvetti M.V. Investigation of the steady engulfment regime in a three-dimensional T-mixer // Phys. Fluids, 2013. Vol. 25. Is. 6. 064102

28. Fani A., Camarri S., Salvetti M.V. Unsteady asymmetric engulfment regime in a T-mixer // Physics of Fluids, 2014. Vol. 26. Is. 7. 074101

29. Galletti C., Roudgar M., Brunazzi E., Mauri R. Effect of inlet conditions on the engulfment mode in a T-shaped micro-mixer // Chem. Eng. J., 2012. Vol. 185. Pp. 300313.

30. Gavrilov A.A., Minakov A.V., Dekterev A.A., Rudyak V.Ya. A numerical algorithm for modeling steady laminar flows of non-Newtonian fluids in annulus with eccentricity // Calculation Techniques, 2012. Vol. 17. Is. 1. Pp. 44-56

31. Gavrilov A.A., Minakov A.V., Dekterev A.A., Rudyak V.Ya. A numerical algorithm for modeling laminar flows in an annular channel with eccentricity // J. of App. and Ind. Math., 2011. Vol. 5. Is. 4. Pp. 559.-568

32. Patankar $S$. Numerical methods in heat transfer and fluid dynamics. M.: Energoatomizdat, 1984.

33. Bystrov Yu. A., Isaev S.A., Kudryavtsev N.A. Leont'ev A.I. Numerical simulation of vortex based enhancement of heat transfer in tube packages. Shipbuilding, 2005.

34. Ferziger J.H., Peric M. Computational methods for fluid dynamics. Berlin: Springer Science+Business Media Inc., 2002.

35. Leonard B.P. A stable and accurate convective modeling procedure based on quadratic upstream interpolation // Comp. Math. Appl. Mech. Eng., 1979. Vol. 19. Is. 1. Pp. 59-98

36. Rhie C.M., Chow W.L. Numerical study of the turbulent flow past airfoil with trailing edge separation // AIAA Journal, 1983. Vol. 21. Is. 11. Pp. 1525-1532

37. Trottenberg U., Oosterlee C.W., Schüller A. Multigrid. Academic Press, 2001.

38. Harr L., Gallagher J.S., Kell G.S. NBS/NRC Steam Tables. Hemisphere Publishing Corp., 1984.

39. Marsh K.N. Ed., Recommended reference materials for the realization of physicochemical properties. Blackwell Scientific Publications, Oxford, 1987.

40. Sengers J.V., Watson J.T.R. Improved international formulations for the viscosity and thermal conductivity of water substance // J. Phys. Chem. Ref. Data, 1986. Vol. 15. Is. 4. Pp. 1291-1312 